\title{
How Dangerous Is Your Mind: Are There Health-Related Risks of Placebo/Nocebo Responses?
}

\author{
Frauke Musial $^{\mathrm{a}}$ Paul Enck ${ }^{\mathrm{b}}$ \\ ${ }^{a}$ National Research Center in Complementary and Alternative Medicine (NAFKAM), Department of \\ Community Medicine, Faculty of Health Sciences, UiT, The Arctic University of Norway, Troms $\varnothing$, Norway; \\ ${ }^{b}$ Department of Internal Medicine VI: Psychosomatic Medicine and Psychotherapy, Medical Faculty, \\ Eberhard Karls University Tübingen, Tübingen, Germany
}

One of NAFKAM's tasks assigned from the Norwegian health authorities is to inform about complementary and alternative treatment. Part of our information assignment is that we receive questions and try to answer them. A couple of weeks ago, there was one particular question that caught my attention: How dangerous is the placebo or nocebo effect? Can we die from it?

And, in fact, a short google search revealed a good mixture of contributions in the popular press, health sites, etc. Some were very serious and well researched, some were sensational and lurid. Nonetheless, most of these were at some point stressing the fact that negative expectations can make you sick.

The problem begins already with the question on how to define placebo/nocebo responses. Often placebo is described as desired health benefits after an inert treatment while nocebo is defined as unwanted health effects after an inert treatment. This definition is a pragmatic one for the purpose of this editorial, but placebo/nocebo research is a steadily growing field, and the understanding of its underlying neurobiological foundations has come a long way [13]. Nonetheless, it is still difficult to find a definition that covers all aspects of the placebo/nocebo phenomenon [4].

One factor that is doubtlessly central in the mediation of placebo/nocebo responses is expectations [5]. Expectations and beliefs have neurobiological consequences: nothing that is in our minds and has a strong emotional valence will be without effect on our bodies. To expect otherwise would be a fallback into the Cartesian way of

\section{KARGER}

(O) 2019 S. Karger AG, Basel thinking that the mind is a separate entity from the body. The expectation of an anticipated positive consequence will trigger the brains' reward system [1,2]. Alongside expectations, conditioning of bodily functions has also been shown to be essential in order to understand the neurobiology of the placebo/nocebo responses. Why is this so? What is the biological relevance of such responses?

The ability to anticipate and prepare is essential for survival. An organism needs to anticipate and prevent harm and likewise to anticipate and utilize situations/responses that are beneficial. Thus, neurobiological changes, such as for example the conditioned immune responses, in Ader and Cohen's [6] original experiments which are mediated through central nervous system mechanisms such as the reward system or the stress/anxiety network, are preparatory responses in order to avoid harm, as in the case of nocebo, or in order to maximize benefit, as in the case of the placebo response [1].

So, can we die of a belief or an expectation? Negative expectations can at least have harmful effects, such as is the case when reading the list of adverse events potentially associated with a therapy and then experiencing them. In the worst case, the anticipation and experience of side effects of a drug may prevent a patient from taking it. And, latest in a situation like that, the expectation of symptom worsening (call that a nocebo effect) induces harmful effects. Nonetheless, it is important to be aware of the fact that it is not the expectation or belief itself that induces the symptoms, it is the behavioral consequences 
of that belief that causes nocebo effects - similar to a selffulfilling prophecy.

One of the consequences can be that people die of diseases and their inadequate treatment, and sometimes even despite adequate treatment; however, it is not the drug, nor doctor, nor fate that is to be blamed, it is the behavioral consequences, often avoidance to take the necessary actions, that is the cause of these fatalities.

Placebo effects - and to some degree also nocebo effects - are not as much for persons with high self-efficacy and an internal "locus of control" (I can handle this, I am in control) but for the insecure patient, those who are in need of an external source of confirmation, be it a physician in a white coat, an alternative practitioner, a proxy, a priest, or a prescription leaflet. But, independent of this, it would be unethical to withhold information about the potential risk of an intervention or a drug, and patients need to be able to make informed choices - if they decide to do so. One possibility to tackle the challenge of inducing nocebo reactions through informed consent is to inform patients about how negative expectations may shape our experiences and thus inoculate them against a nocebo reaction [7]. And if you as a patient know that reading about side effects in the leaflet makes your feeling sick, do not read them but talk to a person you trust, your doctor, your alternative practitioner, or your pharmacist about your concerns. They are obliged to inform you, but they can at the same time also attend to your worries and answer your more specific questions.
Why and how is the placebo/nocebo challenge relevant for complementary and alternative medicine? And why should this topic be of interest for practitioners working in the field? Because the challenge how to navigate between maximizing the placebo response to a treatment while minimizing potential nocebo effects and at the same time be honest about potential adverse events [3] is the same for all health-related professions. An expert consensus on the implications of placebo and nocebo effects for clinical practice has formulated useful recommendations about how to handle that challenge [3]. To us, especially the acknowledgement that placebo effects are part of every treatment, as well as the recommendation to not consider deception as a necessary part of the placebo effect, is almost the most astounding, as placebo is normalized through the acceptance of its existence in all health-related interventions.

The third recommendation asks to ensure a patientclinician relationship that is characterized by trust, warmth, and empathy. Such a relationship is deemed as the most resilient factor in order to protect patients against nocebo effects and to maximize the desirable placebo effects of a treatment. Fortunately, it is exactly that compassionate attitude many health care providers, conventional or alternative, take pride in and strive to implement it in their everyday practice. Thus, maximizing the placebo effect is desirable and, considering our inherent desire as humans to avoid harm and maximize benefit, fundamentally ethical and deeply humane.

\section{References}

1 Enck P, Benedetti F, Schedlowski M. New insights into the placebo and nocebo responses. Neuron. 2008 Jul;59(2):195-206.

2 Wager TD, Atlas LY. The neuroscience of placebo effects: connecting context, learning and health. Nat Rev Neurosci. 2015 Jul;16(7):40318.
3 Evers AW, Colloca L, Blease C, Annoni M, Atlas LY, Benedetti F, et al. Implications of Placebo and Nocebo Effects for Clinical Practice: expert Consensus. Psychother Psychosom. 2018;87(4):204-10.

4 Howick J. The relativity of "placebos": defending a modified version of Grünbaum's definition. Synthese. 2017;194(4):1363-96.

5 Kirsch I, editor. How Expectancies Shape Experience. Washington, DC: American Psychological Association; 1999.
6 Ader R, Cohen N. Behaviorally conditioned immunosuppression. Psychosom Med. 1975 Jul-Aug;37(4):333-40.

7 Klinger R, Flor H. Clinical and Ethical Implications of Placebo Effects: Enhancing $\mathrm{Pa}$ tients' Benefits from Pain Treatment. In: Benedetti F, Enck P, Frisaldi E, Schedlowski M, editors. Placebo. Handb Exp Pharmacol 225. Berlin: Springer; 2014. p. 217-35. 